\title{
Research on the Innovative Approach of Intergrowth of Xipi Lacquer Art in Huizhou and Modern Product Design
}

\author{
Fuliang Sun ${ }^{1}$, Wei Shu ${ }^{1 *}$ \\ ${ }^{1}$ Art School, HuangShan University, HuangShan, AnHui, 245041, China
}

\begin{abstract}
Xipi Lacquer Art in Huizhou was once well-known all over China with the brilliant Huizhou merchants and it was once also annihilated in the river of long history. The surface of Huizhou Xipi Lacquer Art is so bright that it can serve as a mirror, and the swirling, complicated and changing patterns endow it with mysterious and gorgeous beauty. In modern times, since the technique of Huizhou Xipi Lacquer Art recovered all that was lost, its special aesthetic sense of beauty and the craftsman's pursuit of superlative craftsmanship have led to the increasing emphasis on the objects produced by it for people to appreciate. This deviates from the public's demand for stable quality and economic utility, and it is difficult to integrate into the consumer market. In this paper, through the in-depth analysis of the history and context of Huizhou Xipi Lacquer Art, the author combines with the theory of contemporary product design to explore the advantages and disadvantages of Huizhou Xipi Lacquer Art into modern product design. Besides, the author uses the scientific research method to find a breakthrough for the two symbiosis and concludes the approach to realize the innovation and development of the excellent traditional technology in the era of science and technology.
\end{abstract}

\section{Introduction}

Chinese traditional lacquer art is well-renowned. In the long river of history, the lacquer craftsmen give lacquerware profound cultural heritage from generation to generation. Qiangjin（炝金）, gold drawing and other art crafts have a profound impact on Japan and other places, which is a representative of international cultural exchanges. Xipi Lacquer Art is one of the main branches of Chinese traditional lacquer art, which shows the exquisite wisdom of Chinese traditional craft. The unique combination of lines and colors of Xipi Lacquer Art makes it misted, floating and unpredictable, implying the simple concept of mutual integration of man and nature. However, Xipi Lacquer Art, once closely related to people's life, has gone through several divisions of history into periods. Although it has been restored to heritage, most of the Xipi Lacquer Arts are displayed in the exhibition hall in the form of collection, which is gradually far away from our daily life. How to bring this shining traditional handicraft back to modern life is of great significance to the inheritance and development of Xipi Lacquer Art.

\section{The development history and status quo of Xipi Lacquer Art in Huizhou}

The lacquerware in China has already begun to develop in the Neolithic period. At that time, the ancestors had a simple understanding of the performance of the lacquer and they could also use it. In the 7,000-year-old Hemudu culture, the ancestors had painted wooden bowls with red lacquer. By the Song Dynasty, the variety of lacquerware ware was more complete. Storage appliances, cooking utensils, musical instruments, burial objects and daily furnishings are available in all varieties.

\subsection{The history of Xipi lacquer}

Xipi lacquer, also known as tiger skin lacquer, pineapple lacquer, manifest lacquer and changeable painting techniques, is a branch of the traditional lacquer art of the ancient cultural heritage of the Chinese nation, which is highly praised for its mysterious and abstract texture ornamentation. During the Longqing Year of Ming dynasty, Huang Cheng, a native Xin'an of Anhui province, wrote a monograph of lacquering The Records of Lacquer Decoration (figure 1), in which there is a sentence "Xipi lacquer, also known as Western leather or 
Rhinoceros skin. The cloudlet, the round flower and the pine tree skin are its patterns. If you look closely at the

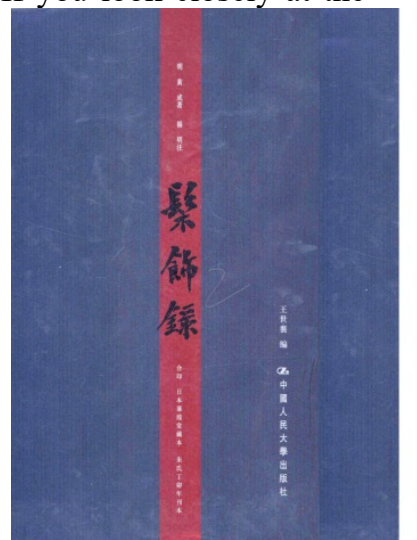

Figure 1. The Records of Lacquer Decoration: Xipi
Xipi lacquer, it will redden and smoothness is regarded as its beauty" [1].

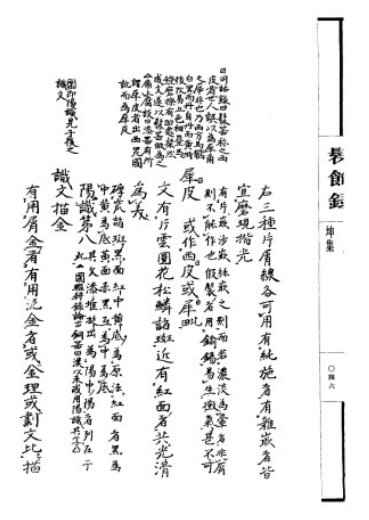

The Xipi Lacquer Art has a long history. The most important version of the origin of its name "Xipi" is the "origination theory of saddle" and "the theory of belt rhinoceros and Xipi". In 1984, the Xipi Huangkou Wineglass of Three Kingdoms Period (figure 2) was excavated in Zhu Ran's tomb in Yushan District, Maanshan, Anhui Province, the body of lacquer says "the surface is black, the middle part is red, the bottom is yellow, and the pattern of Xipi is cloudlet. In the Tang Dynasty, Yuan Jiao and Gan Zeyao mentioned the pillow of Xipi in the volume of 1956 of Taiping Guangji; in the
Song Dynasty, Wu Zimu mentioned in the Mengliang Records that Qi's Xipi shop by the Qinghu River was paratactic with You's lacquer shop, which shows that there was a lacquer workshop mainly to make Xipi. Mr. Wang Shixiang wrote in his book An Interpretation of The Records of Lacquer Decoration that The Old Man of the West Lake Fan Shenglu (the author is Lin'an in the Southern Song Dynasty, and this volume is from the Yongle Canon) mentions Xipi movement, a daily household appliance, which shows that Xipi lacquerware is quite common at that time.

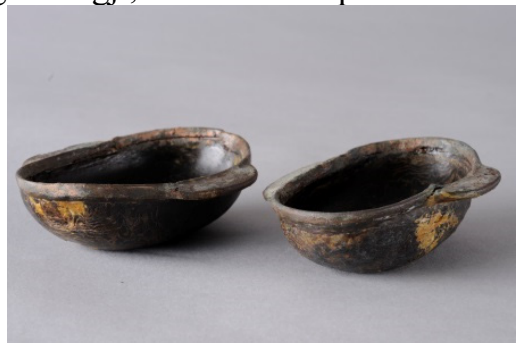

Figure 2. the Xipi Huangkou Wineglass of Three Kingdoms Period

\subsection{Xipi Lacquer Art in Huizhou}

The lacquerware of Huizhou come down in one continuous line with the traditional Chinese lacquer technology, and it is inferred from the historical records that the lacquerware of Huizhou has a history of more than 1000 years. The natural environment of Huizhou is beneficial to the growth of lacquer trees, and Huizhou is rich in high-quality raw lacquer. Since the Song Dynasty, Huizhou has a prosperous lacquer industry, Huizhou lacquerware has unprecedented development to the Ming and Qing dynasties. There are fine lacquer in many cities or places in Huizhou, which was recorded from A History of Illustrious Families in Xin'an of Song Dynasty to A History of Huizhou of in the period of Emperor Daoguang in the Qing Dynasty. During the Ming and Qing dynasties, Huizhou merchants were rich all over the world, which promoted the development of Huizhou culture, art and economy, thus the "lacquerware of Huizhou" could become popular all over the country, of which the most popular one is the Xipi lacquer of Tunxi. In the Qing Dynasty, Xipi lacquer craft was used to make all kinds of utensils, ranging from boxes to furniture, which has a variety of categories and is the best choice for the imperial tribute in the Qing palace.

Huizhou lacquerare ware gradually declined in the late Qing Dynasty, Xipi lacquer, lacquer sand inkstone, mother-of-pearl inlay and other craft which were once world-renowned also declined with Huizhou lacquerare, they almost disappeared. After the founding of the People's Republic of China, Yu Jinhai, a former head of the Technical Unit of the Tunxi Institute of Lacquer Technology and consultant of the Innovation Group, has been engaged in lacquer making and research for many years, excavating the unique handicraft "pineapple lacquer" for nearly 50 years, and resuming the production of "pineapple lacquer" and "lacquer sand inkstone" (Figure 3), which makes Huizhou Xipi lacquer reappear in the world. 


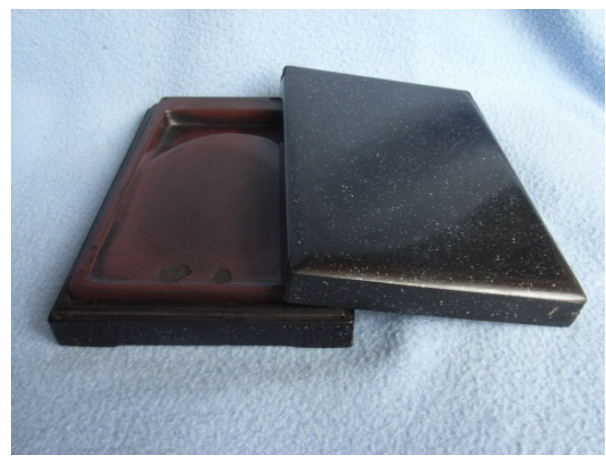

Figure 3. The lacquer sand inkstone made by Mr. Yu Jinhai

\subsection{Status Quo of Xipi Lacquer Art}

Nowadays, "Huizhou lacquerware" is listed as the third batch of intangible cultural heritage protection projects by the state. The representative inheritor of technique, Gan Erke is the representative of Xipi lacquer art in Huizhou. His Xipi lacquer works has its own uniquenessin the painting texture, the objects and textures are combined with the fine and delicate characteristics of the lacquer, which shows the simple and natural beauty of lacquer. Moreover, he pioneered the gold foil into the lacquer, he made red, green, black lacquerware and other lacquerware of a series. Mr. Gan Erke attempted to describe the texture of thousands of images in nature, which has greatly enriched the expressive power of traditional Chinese lacquer language and broken the fixed presentation pattern of conventional lacquerware. His innovative initiatives have pushed the art of Huizhou Xipi lacquer to a totally new level, bringing the creation of Xipi lacquer into a totally new aesthetic field (figure 4).

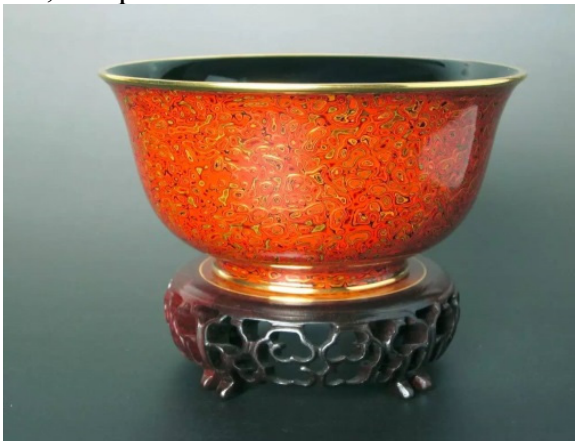

Figure 4. Red and gold spotted Xipi lacquergold reed Ruyi bowl made by Mr. Gan Erke

\section{$3 \quad$ Technological and artistic features of Xipi Lacquer Art in Huizhou}

The pattern of Huizhou Xipi Lacquer Art is very smooth, which is made of lacquers of different colors. The pattern of the surface is like the flowing water, curved water flowing tendons, cloud, round flowers, skin of pine tree and so on. Its pattern changes infinitely, which shows the law of futility, natural flow, brilliant color, people feel it is very beautiful. Compared with other lacquer art, Huizhou lacquer art uses a lot of carrier and the same style of painting, but the texture is very different. Modern scholars and craftsmen start with The Records of Lacquer Decoration, they have a consensus on Xipi Lacquer writing and practical comments. Most of them recognize the creative inspiration and language from nature to enrich its connotation and culture.

\subsection{Technological features of Xipi Lacquer Art in Huizhou}

There are few differences between lacquerware and other traditional lacquerware before finishing lacquering, the key difference is in the process of making a protrusion higher than the surface of the lacquerware. In order to pave the way for the presentation of the final pattern, the protrusions with different sizes and heights are made on the objects by making protrusions higher than the surface of the objects. In the seventh issue of Cultural Relics Reference in 1957, Mr. Yuan wrote in Talk about the Xipi Lacquerware that the way to get Xipi lacquer from the old painters Gui Maokao is to: "mix the mineral yellow into raw lacquer to form a certain consistency to lacquer on the wooden cigarette-bag pole. When it is not dry, gently push the lacquer out of a small, protruding tip with your right thumb, turning from one end of the pole to the other. After pushing, there are some scales like snakeskin. The term for this process is called "making a protrusion higher than the surface of the lacquerware", which means building a high "dam" on the flat ground, like building with soil to resist water. After drying out, put the red and black paint on the top of each protruding spire, about four or five times each time, the purpose of which is to make the tip grow higher. After that, the lacquerware was painted with red and black. Every time the lacquer is painted, it will be put into the shade once. Up to twenty coats of paint can be applied. Finally, polish the lacquerware with millstones and charcoal. Where there is a high rise, after grinding, it will be 
surrounded by a circle of paint layer, showing a pattern similar to pine scales."[2] (Figure 5). Therefore, Wang Shixiang mentioned in An Interpretation of The Records of Lacquer Decoration: "from the above practices, it can be seen that the reason why Xipi lacquer was listed as a door inlaid by Huang's family is that the painted land is low and concave, and the color paint is filled for many times, and finally it is ground out; there are similarities with various practices of the same category."

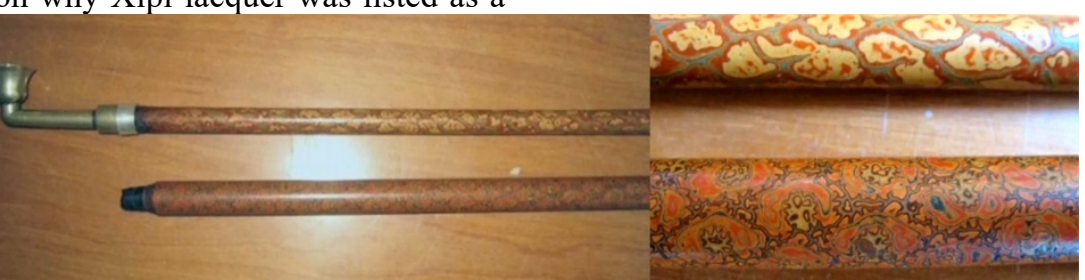

Figure 5. Xipi Lacquer small-bowled long-stemmed (tobacco) pipe

\subsection{The technological process of Xipi Lacquer Art in Huizhou}

Since the art of Huizhou Xipi lacquer reappeared in the world, many outstanding craftsmen have devoted themselves to groping, practicing and innovating. The first thing to consider in the production of Xipi lacquer is how to choose the fetal bone, and the procedure of making different fetal bones is different. For utensils, daily use and decorative arts and crafts, the first choice is the mingle fetal, followed by wood fetal, fur fetal, bamboo fetal, paper fetal, pottery fetal, metal fetal and so on. After making the body, it will be lacquered, damed, painted, sanded, polished [3] (Figure 6). A piece of lacquer ware is often made by craftsmen for months or even years, in which no errors are allowed, or the previous work is lost, which is the precious part of an excellent rhinoceros-skin lacquer ware.

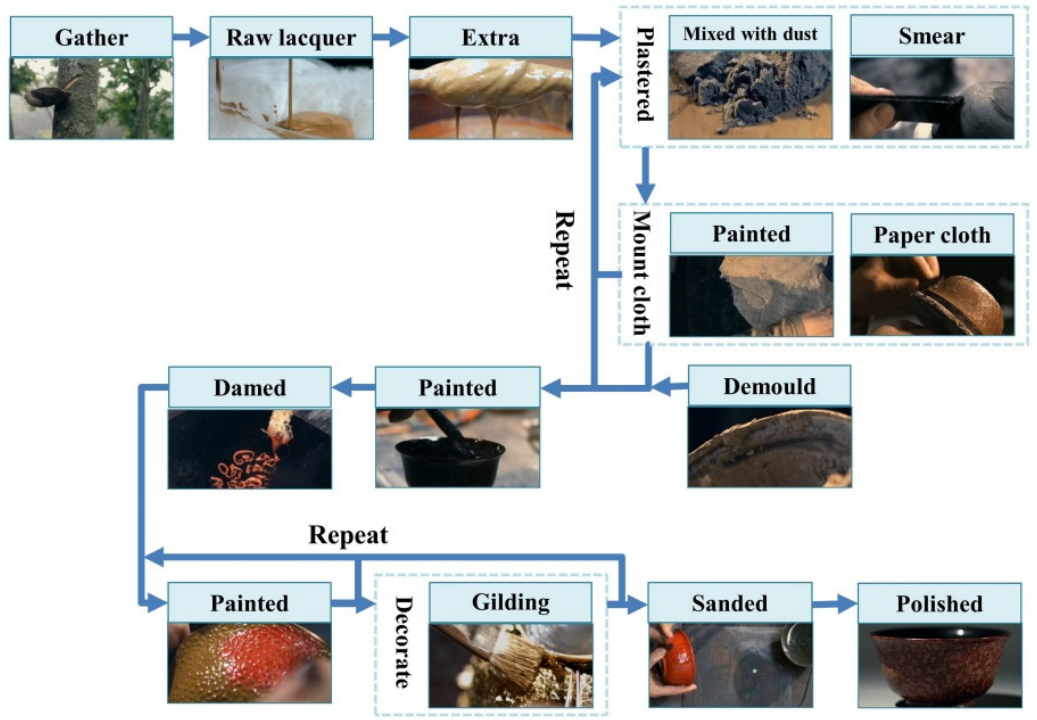

Figure 6. Process flow of contemporary Huizhou Xipi lacquer

\subsection{Artistic characteristics of Huizhou Xipi Lacquer Art}

Huizhou Xipi Lacquer Art adopts a kind of complex and irregular-looking texture to express people's yearning for mysterious and gorgeous beauty, which is to explore the depths of life's desire for beauty. Gui Maokao once mentioned: "the shape of the surface pattern of this kind of lacquerware depends on the method of beating the dam, and the color depends on the paint on each time", which makes the final texture of the Xipi Lacquer occasional and controllable.
3.3.1. Controllability. Controllability refers to "in the development and change of things, there may or may not be situations or situations that can occur in this way or in that way" [4]. The controllability of Xipi Lacquer is mainly due to the size, distance, density or overlay of the dam when the craftsmen hit it in their creations; in addition, in the following processes, the paint is superimposed or cut in an orderly or disorderly manner; the time of painting the paint, the humidity in the air, the temperature of the paint, the degree of thinness of the paint; and the resulting color of the paint and the dam, the random form between the dam and the dam and the paint, and finally the lacquer lute has a rich, free and artistic texture effect (Figure 7). 

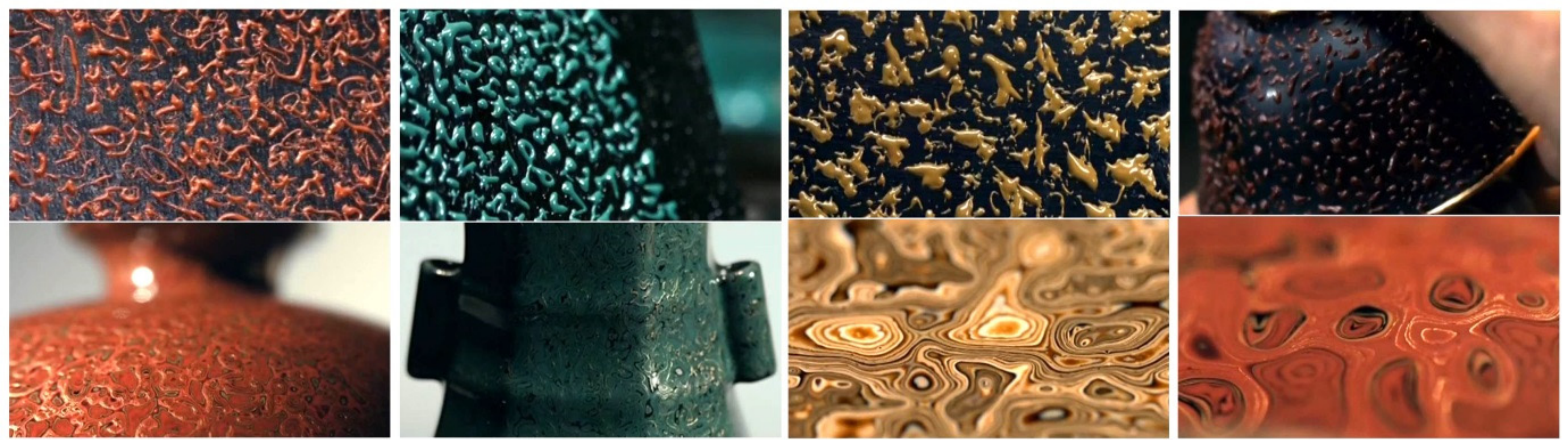

Figure 7. Different ways of digging the dam and the corresponding final texture

3.3.2. Controllability. Controllability refers to "we must first determine whether its state is controllable before designing some optimal control systems" [5]. The controllability of Xipi Lacquer is mainly reflected in that after many tests, the craftsman can control the influence of the thickness of the paint layer, the degree of drying, the humidity and temperature in the air on the overall rhythm of the whole work. When painting, with the help of the planning of the sequence, time and thickness of the painting overlay, the general image of the final pattern of the work is predicted before making. Finally, in the process of "grinding", the craftsman controls the overall tone of the work and adjusts the presentation effect of the final texture pattern by grasping the grinding time and strength. Light grinding, the upper color paint is the main tone; more grinding, the lower color paint will occupy the top; various forms of trend, virtual and solid, dense, deep and shallow, high and low, varied, all depend on the manufacturer to control the intensity of grinding, the most wonderful level in the peak turn, deduce the changeable and multi-terminal texture.

\section{$4 \quad$ The challenge and opportunity of Xipi Lacquer industry in Huizhou}

\subsection{Back to functional}

Huizhou Xipi lacquerware can be classified as practical and appreciative according to its function. Practical types of lacquerware are mainly daily life appliances and stationery, this type of lacquer ware emphasizes practicability. The lacquerware of appreciation class is imitated ceramic, bronze ware and so on ancient shape manufacture. The shape of the utensils is elegant and exquisite, and this kind of lacquer ware takes aesthetic taste and appreciation as the main function. Eventually, they are in the hands of various galleries, galleries, museums or lacquer ware collectors in the form of exhibitions or pure art[6]. Today, Huizhou Xipi lacquerware is still mainly handicraft, the yield is extremely low. They are works of art created by lacquer craftsmen using exquisite rhinoceros lacquer art. Most of the objects to appreciate the main type of lacquer ware, a small number of household goods have been far away from practicality, focusing on aesthetic and artistic.

\subsection{Combining with new technologies}

Today, the market is increasingly open, but the development of Xipi lacquer art in Huizhou is still blind. Most of the craftsmen, as they have done in the past, have tried their best to hone and promote their craft[7]. From the history we can see that the era of lacquer art and utility is also the period of flourishing technology. How to let Huizhou Xipi lacquerware come out of the simple appreciator and get the daily consumer market is our biggest challenge at present. With the progress of science and technology, the mode of production is constantly innovating, and the processing methods and ideas of many traditional technological fields and modern industrialization are accelerated to seek the innovation and breakthrough suitable for the times (Figure 8).

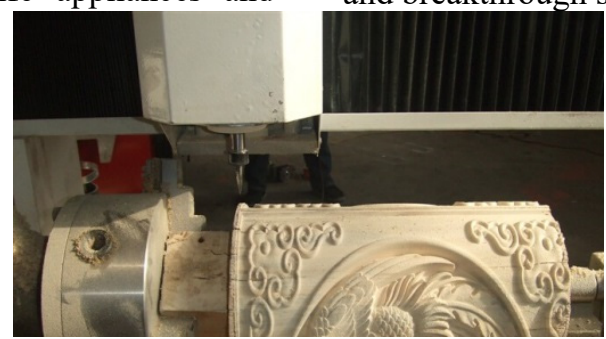

Figure 8. CNC machining equipment integrated into wood carving industry

\subsection{Focus on user needs}

In order to get out of the traditional Xipi lacquer art, we must find the way of production in accordance with the background of the times and return to the essence of practical objects. Now the social product is very rich, people begin to care more about the cultural connotation of consumer goods. Huizhou Xipi lacquer has experienced the quenching of time, as a characteristic cultural carrier to record the vicissitudes of life, it has a unique charm [8]. We hope to use modern design means, based on practical objects, dig deep into Huizhou traditional rhinoceros lacquer ware technology, find its possible connection point with modern production mode, design a new era rhinoceros lacquer products that meet 
the practical, aesthetic and cultural needs of contemporary consumers through the collision of modern and traditional. And then achieve the purpose of inheriting folk traditional skills, protecting intangible cultural heritage and innovating national culture.

\section{The advantages and exploration of the symbiosis of Huizhou Xipi lacquer and modern products}

The original intention of Huizhou Xipi lacquer ware is to take from citizen and used in citizen. There are many parts of the processes and practices that traditional craftsmen practice to make perfect, habitual or commonplace. Modern designers should constantly reflect, ask questions and awaken in the process of innovation of "daily use is the way"[9], which makes the traditional Huizhou Xipi lacquer gradually break through the simple craft, innovate the development from the industrialization mode of the whole process of design, production and consumption, and form the ecological chain structure of the whole linkage. Only with proper reflection and questioning, can we awaken the future direction, adjust in the exploration, and explore its connection with the times, audience, design, production, health and other factors through the increasingly deep cognition of rhinoceros skin paint.

\subsection{Aesthetic advantages}

The surface color of Huizhou Xipi lacquer presents free texture pattern. From the paint, we can see that it is elegant, which is consistent with modern aesthetics and can be directly used in modern product design.

The greatest feature of the Xipi lacquer is that all its designs are not consciously depicted, but the craftsmen show the unconsciousness of the hills in their hearts, as if injecting the soul into them. This makes the appearance of rhinoceros paint associated with modern design, its texture design ideas and modern graphic reconstruction theory, bionic design philosophy, design ideas. Traditional rhino lacquers are used as such to decorate weapons, tools, furniture, ornaments, household utensils and other practical appliances[10] (Figure 9); their common label patterns, such as cloud, pine scales, round flowers and pineapples, are natural and are typical bionic symbols; these compositions, textures and colors can be used directly in the design of modern product graphics, colors and symbols to give people an intangible, natural and vivid space for imagination (Figure 10).

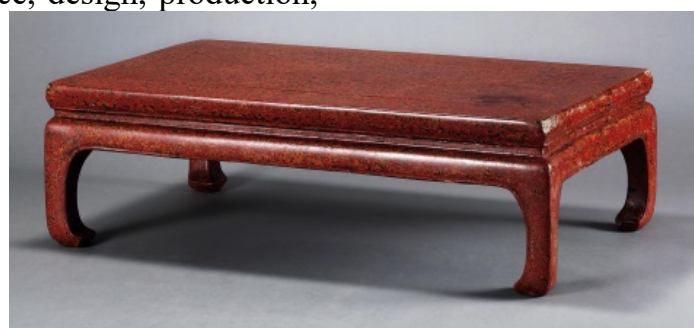

Figure 9. The Middle of Qing Dynasty Xipi lacquer rectangle tea table

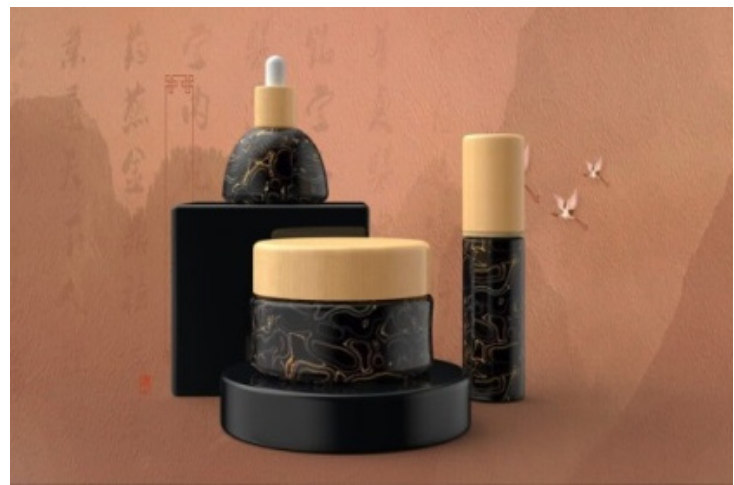

Figure 10. Xipi lacquer design of cosmetic outer package

\subsection{Technological advantages}

The tools used in the processing of Huizhou Xipi lacquer are very primitive, which provides a wide space for modern tool intervention. In the production process, the core processes such as making a dam and painting mostly adopt the free coating method, which is conducive to the tool intervention to improve the production efficiency, but also to maintain the individual differences of products.

The production of the traditional Huizhou Xipi lacquer ware is extremely focused on the craftsman's "craft beauty"[11], the tools used are very original (figure 11), which makes the production inefficient and the production extremely low. In modern society, it is hard, as in the past, to produce practical lacquer ware for daily consumption through the manual work of a large number of painters. And the ordinary rhino lacquer utility will not pursue the ultimate "beauty of craftsmanship" like purely appreciative lacquer ware. Tool intervention can effectively improve production efficiency, traditional craftsmen will also innovate the choice of tools to use modern materials, such as the use of various modern grinding tools to complete the grinding. With the scientific recognition of the great lacquer and the 
constant exploration of it, the number of tools available is growing with the support of modern technology[12], and even the most conservative lacquer craftsmen will be impressed with the modern industry's lacquer products in the foreseeable future.

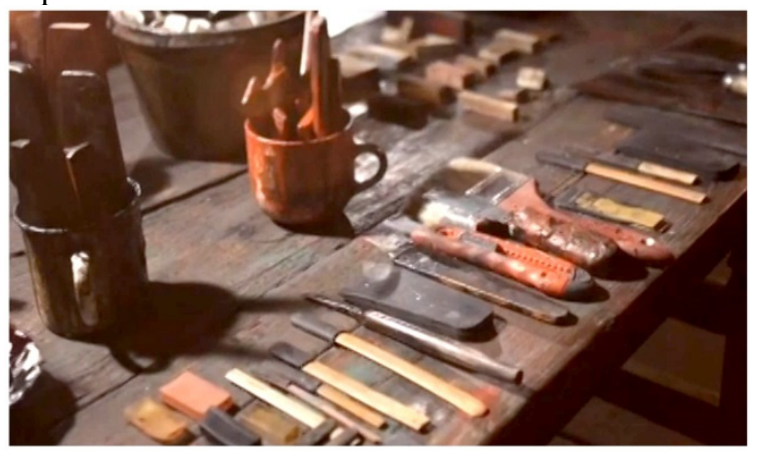

Figure 11. Xipi lacquer traditional tools

\subsection{Material advantage}

The main material of Huizhou Xipi lacquer is lacquer, which is a kind of natural resin paint. It belongs to pure natural material and fits the concept of green design.

The main material of Huizhou Xipi lacquer is natural lacquer cut from lacquer tree by hand. It has the reputation of corrosion resistance, strong acid resistance, strong alkali resistance, moisture resistance, insulation, high temperature resistance, soil resistance and so on. The forefathers recognized the performance of lacquer from the Neolithic period, and lacquer ware was a common everyday object from business through the Ming and Qing dynasties (Figure12). In the process of modern product design, we cannot only care about their artistry, technology and economy, but also put the concept and method of ecological design into it to pay attention to the sustainable development of human society. As far as the modern design of Huizhou rhinoceros lacquer ware is concerned, if we want it to be re-entered into practical consumer goods (Figure13), we must take seriously the responsibility of the individual product of lacquer ware and the survival, health and ecology of the whole society.

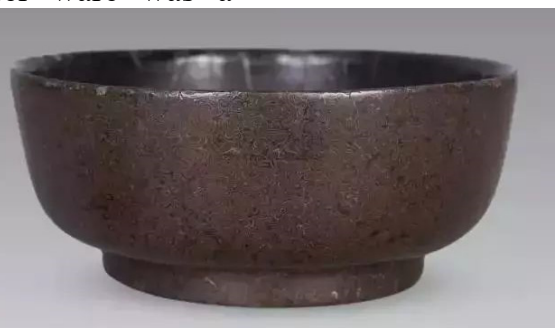

Figure 12. Qing Dynasty Xipi lacquer bowl

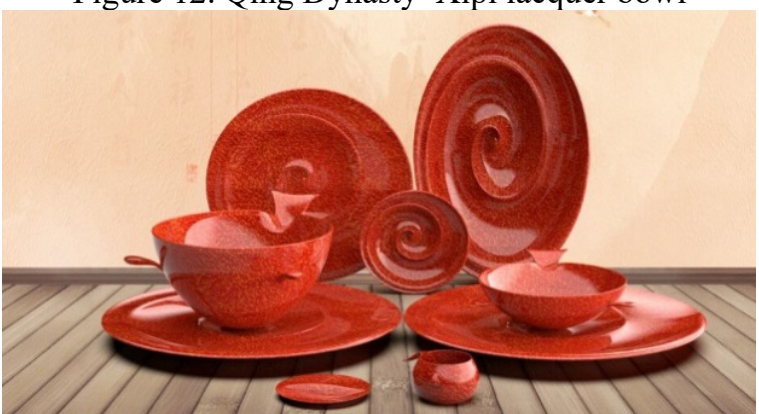

Figure 13. The design of Xipi lacquer tableware

\subsection{Cultural advantages}

The regeneration of Huizhou Xipi lacquer art can make us deeply feel the love, inheritance determination and rejuvenation of Huizhou craftsmen for Chinese traditional lacquer art.

The expression carrier, form, characteristic and thought of Huizhou Xipi lacquer reflect the brand of fit times, bearing the long history and culture of Huizhou, the nationality and the sense of history contained in it is reflected from instinct and nature. During his visit to the cultural and artistic circles and members of the social sciences of the second session of the 13th CPPCC, President Xi Jinping stressed the need to strengthen cultural self-confidence, grasp the pulse of the times, listen to the voice of the times, adhere to the same pace as the times, take the people as the center, devote the people with fine products, and lead the fashion with Mingde. It is necessary to realize the cultural rejuvenation to realize the national rejuvenation. Huizhou Xipi lacquerware is the model of Huizhou culture. The artist of Xin'an painting directly influences the creation of Huizhou Xipi lacquer from the artistic thought of nature: the color in Huizhou's natural scenery becomes the source of the color of Huizhou Xipi lacquer; the 
surface texture of Huizhou Xipi lacquer works absorbs the expression technique of Xin'an painting school; like the artistic conception rendered in the landscape painting of Xin'an painting, it is fresh and elegant, and pays attention to the beauty with its nature. In our design of Huizhou Wenchuang products, Xipi lacquer is the best annotation (Figure14).

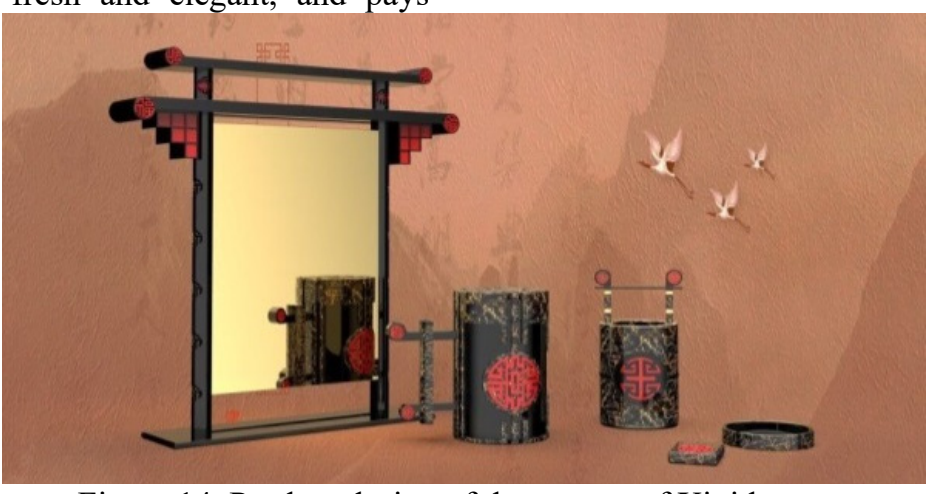

Figure 14. Product design of the pattern of Xipi lacquer

Throughout history, Chinese lacquer art also appeared a period of depression. Compared with us, the Japanese lacquerware craft can flourish continuously because the Japanese craftsmen can grasp the characteristics of their own national culture and have good innovation. China has a good soil for the development of lacquerware technology, continuous innovation can give the vitality of the vigorous development of lacquerware technology. We are in a time of change, with the help of modern design to find the unique expression methods for Huizhou Xipi lacquerware, at the same time, integrating the distinct national elements, combining with the aesthetic philosophy of the times, clearly approaching the position of the market and the public life, integrating the innovative products of Huizhou Xipi lacquerware into people's lives, and promoting the innovation development and cultural revival of Huizhou Xipi lacquer.

\section{Difficulties and measures of symbiosis between Huizhou Xipi lacquer and modern products}

\subsection{Empiricism}

In the same way, there are many difficulties in the coexistence of Huizhou Xipi lacquerware and modern products. Through the unpredictable texture pattern, Huizhou Xipi lacquer gives the work life surprise, and also increases people's curiosity about the rhinoceros lacquer. The surest way to satisfy our curiosity is scientific research methods.

\subsection{E.g drying conditions}

Through the deep exploration of the practical process of Huizhou Xipi lacquer, we find that the creation of Huizhou Xipi lacquerware is very critical to the time, season, climate and even craftsman. These factors are regarded as "natural achievement" in the eyes of traditional craftsmen, which is due to the lack of scientific observation and record of the process of lacquer painting, and simply rely on "experience" to solve their difficulties. Artisans in the "theory of experience" described the painting of large paint when the weather is too hot or too cold, the environment is too dry or too wet not easy to dry. The scientific method is described as follows: the drying of large paint is through the laccase in the paint to make the laccolol in the air oxidation polymerization, so that the paint dry film; There are two main factors that affect the activity of laccase: first, laccase has obvious thermal sensitivity, which is the most active when temperature is $40^{\circ} \mathrm{C}$ and humidity is about $80 \%$. The second is the acid value $(\mathrm{pH})$ of laccase, when the $\mathrm{pH}$ value is 6.7 , the vitality is the largest. With the support of scientific data, people set up clean dust-free, non-ventilated, room temperature control at $15^{\circ} \mathrm{C} \sim 25^{\circ} \mathrm{C}$, relative humidity kept at about $80 \%$ of the special room- "shady room" to adapt to the drying characteristics of large paint. Raw paint coating in this "shady room" drying time is usually 12-24 hours.

\subsection{E.g. color control}

In addition, we can use scientific methods and modern equipment to solve many "empiricism" difficult to overcome problems. For example: according to the modern industrial color standard to build paint color system, and with the help of modern color mixing equipment to maintain the consistency of color; rely on modern enterprise management methods for product quality control, set up a clean and controlled workshop, improve dust-free operation procedures, scientific methods to reduce product defects.

\section{Conclusion}

Huizhou Xipi lacquer art has a profound connotation, which contains various cultures and feelings. The warmth of the craft can not be replaced by the high-tech modern industrial production technology, but we can use the new technology and scientific methods to help Huizhou Xipi lacquerware return to the origin of the utility, and reenter people's daily life. Therefore, through the design of 
the whole life cycle of Huizhou Xipi lacquerware, we explore the way of its symbiosis with modern products, so that this strong culture into modern products, so that consumers feel the flavor of traditional crafts more personally.

In recent years, with the rejuvenation of the Chinese nation and cultural self-confidence, more and more excellent traditional technology has entered people's eyes. To protect these traditional processes, what we need to do is not only remember the techniques of the traditional processes, but also to study them scientifically and to innovate them. Search for the new way out of traditional craft, explore new development space, look for the opportunity of revival. Let's enable it to break through the limitations of traditional crafts, in the new era of inheritance.

\section{Fund}

Research on the Development and Design of Huizhou Cultural Creative Tourism Commodities, Key Project of Humanities and Social Sciences $\mathrm{s}$ of Colleges and Universities in AnHui, Liang Jun, SN: SK2018A0392

\section{References}

1. Huang, C.( Ming Dynasty) The Records of Lacquer Decoration. Unknown, Unknown.

2. Wang, S.(2013) An Interpretation of The Records of Lacquer Decoration. Life Reading Xinzhi Sanlian bookstore. Beijing.

3. Liu, Y. (2019) Research on the development and performance of Xipi lacquer art. Hangzhou Normal University, Hangzhou.

4. Liu, S. (2019) Research on Xipi lacquer art. Hubei University of technology, Wuhan.

5. Li, C. (2019) Application and innovation of Xipi lacquer art in modern lacquer painting. Anhui Normal University, Wuhu.

6. Yue, J. (2017) Research on the production technology and artistic characteristics of Huizhou lacquerware . Anhui University, Hefei.

7. Jin, Y. (2017) Research on Xipi lacquer. Jiangxi Normal University of science and technology, Nanchang.

8. Guo, L. (2014) Study on the current situation of Chinese rhinoceros lacquer. Shanxi Normal University, Xi' an

9. Gan, E., Li, Z. (2019) Gan Erke' talk on the inheritance and development of Chinese lacquerware. Chinese art, (03): 76-81

10. Feng, J., Gan, E. (2010) Perfect lacquerware, craft surpassing Qianlong period. Huizhou Social Sciences, (3): 57-59

11. Liu, Y. (2016) The implication of the art of xipi lacquer in Huizhou. Journal of Zunyi Normal University, 18 (05): 146-150

12. Zhang, Z. (2013) Enlightenment of Japanese lacquer art vigorous development on China. Journal of Hubei second normal university, 30 (11): 77-80 